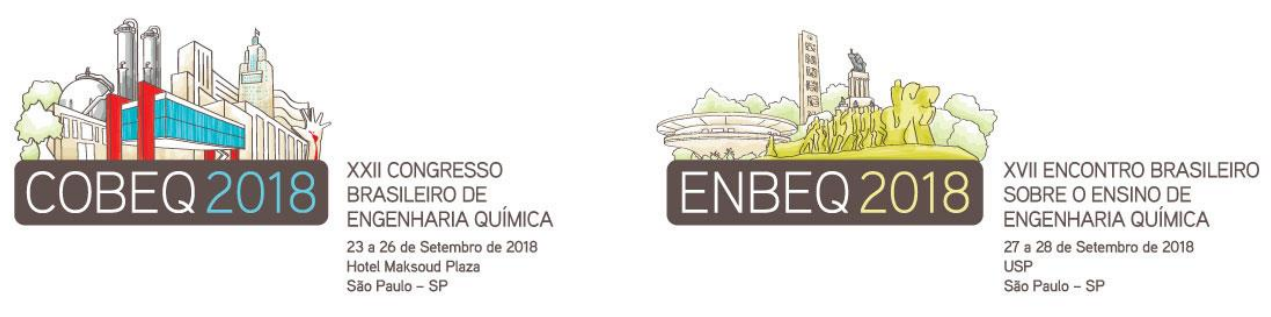

\title{
AVALIAÇÃO DA SELETIVIDADE DE CARBONOS ATIVADOS COMERCIAIS A PARTIR DAS SUAS PROPRIEDADES TEXTURAIS
}

\author{
SANTOS BF ${ }^{1}$, MOURA KO ${ }^{1}$, SIQUEIRA RM ${ }^{1}$, VILARRASA-GARCIA E ${ }^{1}$, \\ BASTOS-NETO $\mathrm{M}^{1}$ e AZEVEDO DCS ${ }^{1}$ \\ ${ }^{1}$ Universidade Federal do Ceará, Centro de Tecnologia, Departamento de Engenharia Química \\ E-mail para contato: diana@gpsa.ufc.br
}

\begin{abstract}
RESUMO - A seletividade de três carbonos ativados comerciais (CA1, CA2 e CA3) foi determinada a partir do modelo de Sips e avaliada em função das propriedades texturais. Estas foram obtidas a partir de isotermas de $\mathrm{N}_{2}$ e $\mathrm{CO}_{2}$ a $77 \mathrm{~K}$ e $273 \mathrm{~K}$, respectivamente, que confirmaram que estes carbonos são materiais altamente microporosos. Isotermas de adsorção de $\mathrm{N}_{2}, \mathrm{CO}_{2}$ e da mistura $\mathrm{CO}_{2} / \mathrm{N}_{2}$ a $298 \mathrm{~K}$ foram adquiridas por método gravimétrico, indicando que os materiais apresentam um comportamento similar. Uma mais baixa seletividade ao $\mathrm{CO}_{2}$ para o CA3 foi observada em mais baixa pressão, sugerindo dependência com a menor quantidade de poros na faixa de 3-5.
\end{abstract}

\section{INTRODUÇÃO}

O dióxido de carbono é um gás nocivo à atmosfera terrestre, sendo um dos principais gases responsáveis pelo efeito estufa. Por isso, nos últimos anos seu processo de captura tem sido amplamente estudado. Para este fim existem diversas tecnologias, dentre as quais podemos destacar a adsorção, que tem como principais vantagens a reversibilidade e baixo custo energético. Nesse meio, os carbonos ativados mostram-se adsorventes promissores por sua alta área superficial e microporosidade (Marsh, 2006).

Dados de equilíbrio de adsorção são de grande relevância para o entendimento do processo de adsorção. A partir deles pode-se determinar a seletividade, que é um dos quesitos fundamentais pra escolha do adsorvente mais adequado para cada aplicação. Dessa forma, neste trabalho, a seletividade de três carbonos ativados comerciais foi avaliada em função das propriedades texturais, em especial a distribuição de tamanhos de poros, determinada pela

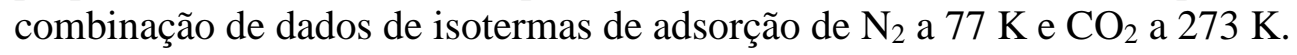

\section{MATERIAIS E MÉTODOS}

\subsection{Materiais}

Para este trabalho, três diferentes carbonos ativados comerciais, denominados como CA1, CA2, e CA3, foram utilizados. 


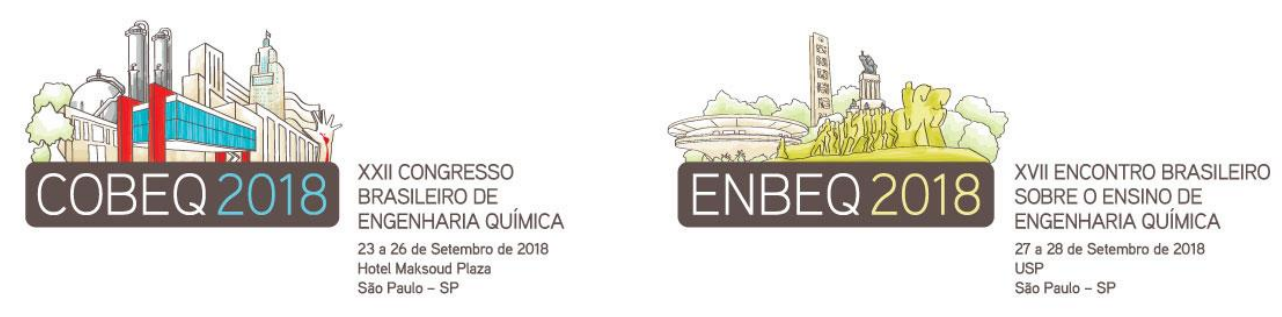

\subsection{Caracterização Textural}

As propriedades texturais das amostras foram avaliadas a partir de isotermas de $\mathrm{N}_{2} \mathrm{e}$

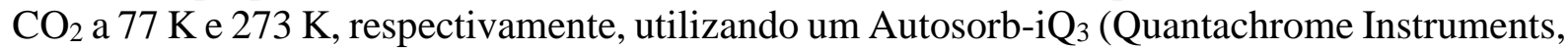
EUA). A partir das isotermas de $\mathrm{N}_{2}$, determinou-se a área superficial específica ( $\mathrm{A}_{\mathrm{BET}}$ ) através da equação de Brunauer-Emmett-Teller (BET), o volume total de poros ( $\mathrm{V}_{\mathrm{P}}$ ) a partir do volume adsorvido em $\mathrm{P} / \mathrm{P}_{0}$ igual, aproximadamente, a 0,95 e o volume de microporos ( $\mathrm{V}_{\mathrm{DR}}$ ) pelo método de Dubinin-Radushkevich (DR) (Rouquerol et al., 2014). A distribuição de tamanho de poro (PSD) foi obtida usando as isotermas de $\mathrm{N}_{2}$ e $\mathrm{CO}_{2}$ aplicando o modelo NLDFT (NonLocal Density Functional Theory) para poros de tipo fenda.

\subsection{Isotermas de Equilíbrio de Adsorção de $\mathrm{CO}_{2}$}

Isotermas de adsorção de $\mathrm{N}_{2}, \mathrm{CO}_{2}$ e da mistura $\mathrm{CO}_{2} / \mathrm{N}_{2}$ a 298,323 e $348 \mathrm{~K}$ foram obtidas por método gravimétrico utilizando uma balança de suspensão magnética (Rubotherm, Alemanha). As amostras foram pré-tratadas por $6 \mathrm{~h}$ a $150{ }^{\circ} \mathrm{C}$ sob vácuo $\left(10^{-3} \mathrm{bar}\right)$. De forma a complementar estes dados, isotermas de $\mathrm{CO}_{2}$ a mais baixa pressão (entre 0-1 bar) foram obtidas utilizando um Autosorb-iQ $\mathrm{Q}_{3}$. Esses dados foram avaliados com o uso do modelo de Sips.

\section{RESULTADOS E DISCUSSÃO}

\subsection{Caracterização Textural}

Os carbonos ativados selecionados foram utilizados sem qualquer modificação prévia. As isotermas de adsorção e dessorção de $\mathrm{N}_{2}$ a $77 \mathrm{~K}$ apresentam um comportamento similar, típico de carbonos ativados microporosos (isotermas do tipo I de acordo com a classificação IUPAC (Thommes et al., 2015)). A Tabela 1 resume as propriedades texturais dos materiais sob estudo, obtidas a partir destas isotermas. Pode-se observar que todos eles apresentam altos valores de área superficial $\left(\mathrm{A}_{\mathrm{BET}}>800 \mathrm{~m}^{2} \mathrm{~g}^{-1}\right)$ e elevada microporosidade $(>80 \%)$. Esses resultados indicam que tais materiais poderiam ser potencialmente bons candidatos para a adsorção de $\mathrm{CO}_{2}$.

Tabela 1 - Propriedades texturais obtidas através das isotermas de $\mathrm{N}_{2}$ a $77 \mathrm{~K}$.

\begin{tabular}{ccccc}
\hline Amostra & $\begin{array}{c}\text { ABET } \\
\left(\mathbf{m}^{\mathbf{2}} \mathbf{g}^{-1}\right)\end{array}$ & $\begin{array}{c}\mathbf{V P} \\
\left(\mathbf{c m}^{\mathbf{3}} \mathbf{g}^{-\mathbf{1}}\right)\end{array}$ & $\begin{array}{c}\text { VDR } \\
\left(\mathbf{c m}^{\mathbf{3}} \mathbf{g}^{-\mathbf{1}}\right)\end{array}$ & Microporosidade \\
\hline CA1 & 1019 & 0,47 & 0,44 & $93 \%$ \\
\hline CA2 & 818 & 0,39 & 0,35 & $92 \%$ \\
\hline CA3 & 1012 & 0,45 & 0,36 & $80 \%$ \\
\hline
\end{tabular}

Na Figura 1 são mostradas as PSD obtidas a partir das isotermas de $\mathrm{N}_{2}$ a $77 \mathrm{~K}$ e complementadas com as de $\mathrm{CO}_{2}$ a $273 \mathrm{~K}$. Nelas observa-se que as PSDs de $\mathrm{CO}_{2}$ revelam picos expressivos em regiões anteriormente não visualizadas nas PSDs de $\mathrm{N}_{2}$, as quais se apresentaram muito semelhantes. Estes picos aparecem na faixa de 3-5 $\AA$. Isso pode ser explicado pelo fato do diâmetro cinético do $\mathrm{CO}_{2}(3,3 \AA)$ ser inferior ao do $\mathrm{N}_{2}(3,64 \AA)$, o que 

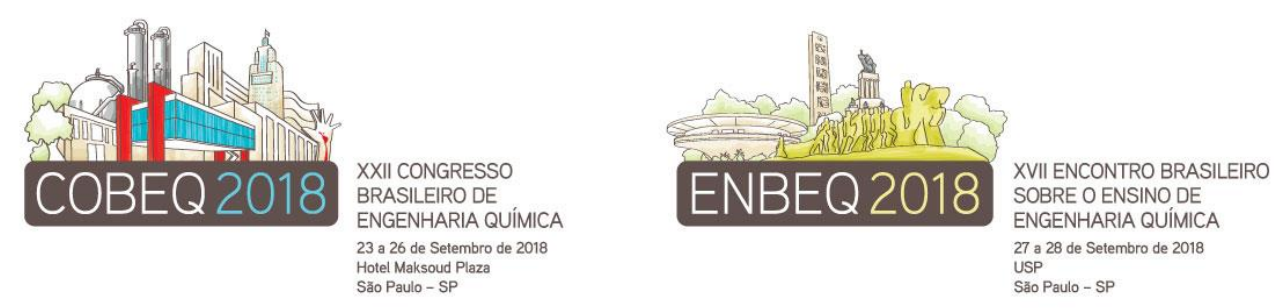

dificulta o acesso do $\mathrm{N}_{2}$ a poros de diâmetro inferior à $5 \AA$ A . Além disso, o $\mathrm{CO}_{2}$ tem um momento quadrupolar que induz a interação entre o gás e a superfície do material (Yang, 2003) e ainda possui uma maior temperatura crítica $(304,35 \mathrm{~K})$ em relação ao $\mathrm{N}_{2}(126,00 \mathrm{~K})$ que favorece a adsorção desse gás.
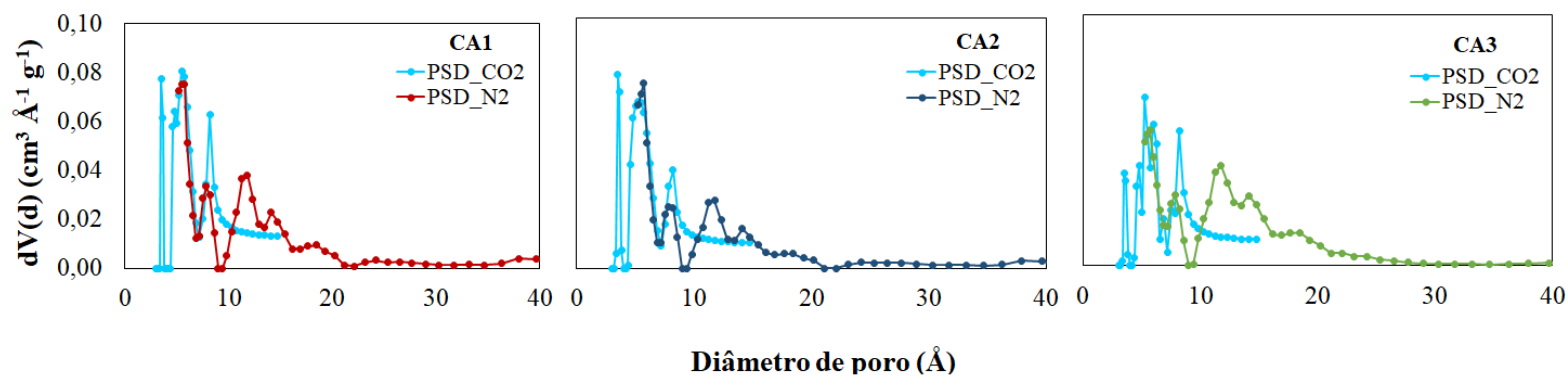

Figura 1 - Distribuições de tamanho de poro obtidas através de isotermas de $\mathrm{N}_{2}$ a $77 \mathrm{~K}$ e $\mathrm{CO}_{2}$ a $273 \mathrm{~K}$ por NLDFT.

\subsection{Isotermas de Equilíbrio de Adsorção de $\mathrm{CO}_{2}$}

A Figura 2 apresenta as isotermas de adsorção de $\mathrm{CO}_{2}$ a $298 \mathrm{~K}$ das amostras obtidas no Autosorb-iQ $\mathrm{Q}_{3}$ (A) e pela balança de suspensão magnética (B). Pode-se observar na Figura 2 (A) que a capacidade de adsorção de $\mathrm{CO}_{2}$ destes materiais segue a ordem CA1 > CA2 > CA3 na faixa de pressão de 0.001 a 1 bar. Por outro lado, a capacidade de adsorção deste gás é similar para todas as amostras em mais altas pressões (Figura 2 (B)).
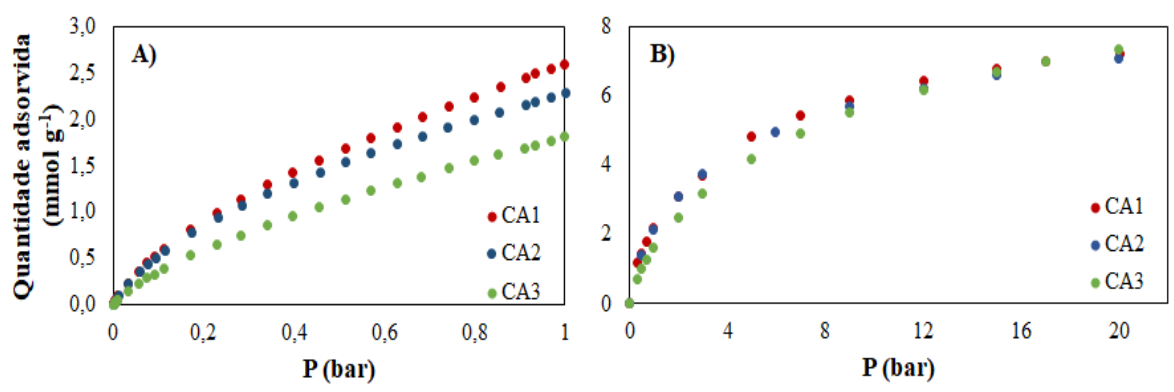

Figura 2 - Isotermas de $\mathrm{CO}_{2}$ a $298 \mathrm{~K}$ obtidas no Autosorb-iQ 3 (A) e na balança de suspensão magnética (B).

A Figura 3 apresenta uma comparação da capacidade de adsorção de $\mathrm{N}_{2}, \mathrm{CO}_{2}$ e da mistura $\mathrm{CO}_{2} / \mathrm{N}_{2}(15 / 85 \mathrm{v} / \mathrm{v})$ a $298 \mathrm{~K}$ para cada um dos materiais. A partir desses dados, e utilizando o modelo de Sips, a seletividade do $\mathrm{CO}_{2} / \mathrm{N}_{2}$ para estes carbonos foi obtida para a faixa de pressão de até 10 bar. De acordo com a Figura 3, pode-se dizer que o carbono CA3 apresenta, em pressões mais baixas, menor seletividade em comparação com os demais carbonos. Estes resultados podem ser explicados pela menor microporosidade observada no carbono CA3 (Tabela 1) e, especialmente, pela presença de menor quantidade de poros de tamanho inferior a $5 \AA$ (Figura 1, PSDs $\mathrm{CO}_{2}$ ). De acordo com Wahby et. al (2012), a presença desses poros estreitos $\left(<5 \AA\right.$ ) é um fator determinante na adsorção de $\mathrm{CO}_{2}$, o que esclarece a maior capacidade de adsorção de $\mathrm{CO}_{2}$ e seletividade a baixas pressões (Figura 2 (A)) do CA1 e CA2 em comparação com CA3. A presença da microporosidade estreita explica também os 

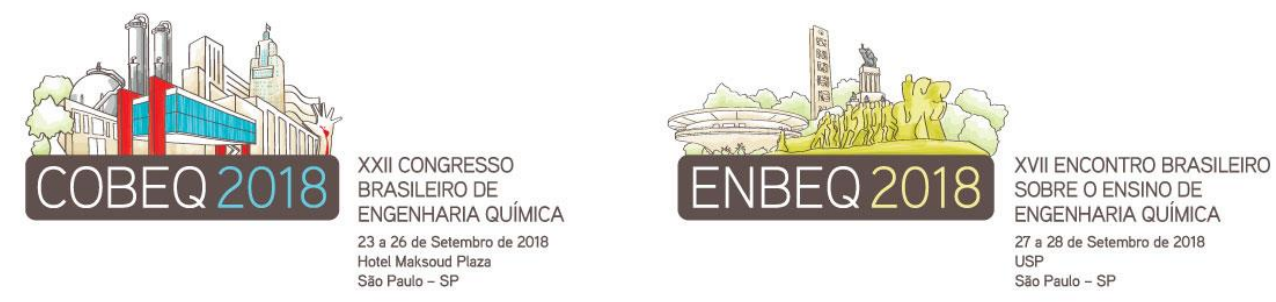

maiores valores de calor isostérico de adsorção calculados a partir das isotermas de $\mathrm{CO}_{2}$ a 298, 323 e 348 K utilizando a Eq. de Clausius-Clapeyron para estes carbonos.
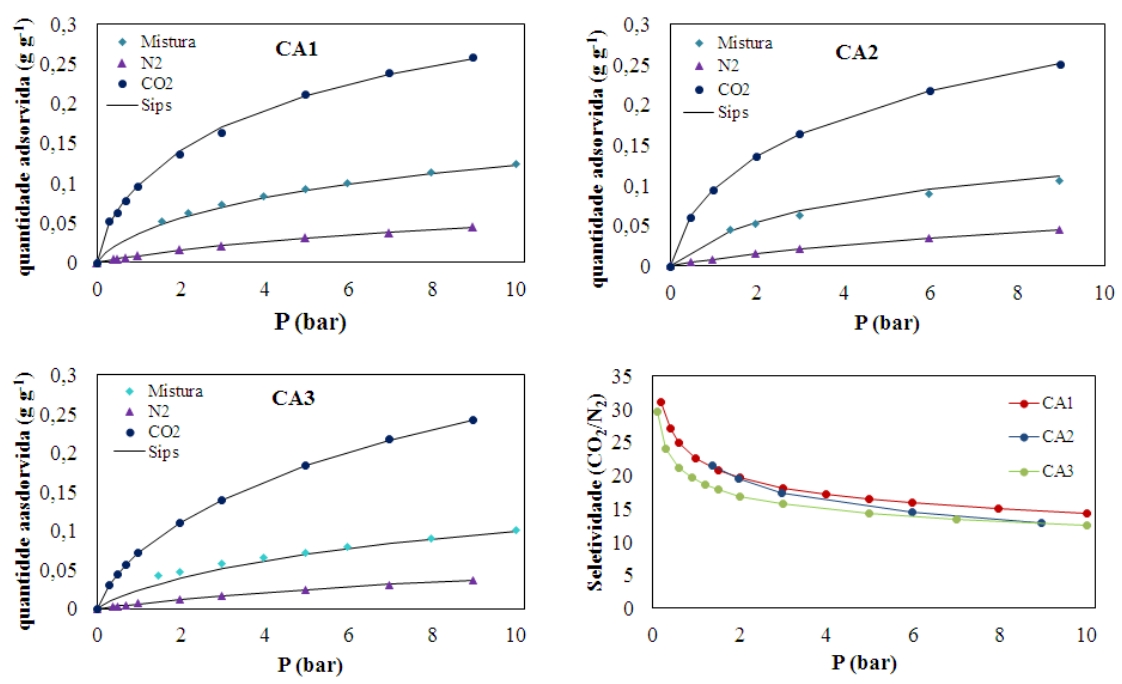

Figura 3 - Isotermas de adsorção de $\mathrm{N}_{2}, \mathrm{CO}_{2}$ e da mistura $\mathrm{CO}_{2} / \mathrm{N}_{2}$ a $298 \mathrm{~K}$ para todos os carbonos ativados e sua respectiva seletividade.

\section{CONCLUSÃO}

Isotermas de adsorção de $\mathrm{N}_{2}$ a $77 \mathrm{~K}$ mostraram que os carbonos apresentam alta área superficial bem como altos valores de volume total de poros e microporos. Isotermas de $\mathrm{CO}_{2} \mathrm{a}$ $273 \mathrm{~K}$ revelaram a presença de maior quantidade de microporos estreitos $(<5 \AA$ ) nos carbonos CA1 e CA2 o que explica a maior capacidade de adsorção destes sobre o CA3 a baixas pressões (0.001-1 bar), assim como a maior seletividade $\mathrm{CO}_{2} / \mathrm{N}_{2}$ observada. Considerando os valores de mais baixas pressões, a seletividade apresenta a ordem CA1 $\approx \mathrm{CA} 2>\mathrm{CA} 3$. Esses resultados revelam que a performance do adsorvente na adsorção de $\mathrm{CO}_{2}$ está estritamente relacionada a uma alta microporosidade bem como à presença expressiva de microporos estreitos, gerando um potencial adequado para a retenção da molécula de $\mathrm{CO}_{2}$.

\section{REFERÊNCIAS}

MARSH H, RODRÍGUEZ-REINOSO F. Activated Carbon. London: Elsevier, 2006.

ROUQUEROL F, ROUQUEROL J, SING KSW, LLEWELLYN P, MAURIN G. Adsorption by Powders and Porous Solids. $2^{\mathrm{a}}$ ed. London: Academic Pres, 2014.

THOMMES M, KANEKO K, NEIMARK A V, OLIVIER J P, RODRÍGUEZ-REINOSO, ROUQUEROL J, SING K S W. Physisorption of gases, with special reference to the evaluation of surface area and pore size distribution (IUPAC Technical Report). Pure Appl. Chem,. v. 87, p. 10511069, 2015.

YANG RT. Adsorbents: Fundamentals and Applications. United States: John Wiley \& Sons, 2003.

WAHBY A, SILVESTRE-ALBERO J, SEPÚLVEDA-ESCRIBANO A, RODRÍGUEZ-REINOSO F. $\mathrm{CO}_{2}$ Adsorption on Carbon Molecular Sieves. Microporous and Mesoporous Materials. v.164, p. 280287, 2012. 